\title{
The effects of genetic background on the island-specific control of a colour polymorphism in Theridion grallator (Araneae: Theridiidae), the Hawailan happy- face spider
}

\author{
G. S. OXFORD* \& R. G. GILLESPIE $\dagger$ \\ Department of Biology, University of York, PO Box 373, York YO1 5YW, U.K. and †Center for Conservation Research \\ and Training, University of Hawaii, 3050 Maile Way, Gilmore 310, Honolulu, HI 96822, U.S.A.
}

\begin{abstract}
The Hawaiian happy-face spider, Theridion grallator, is endemic to four islands in the Hawaiian archipelago. In all populations it exhibits an exuberant colour and pattern polymorphism involving both the carapace and opisthosoma (abdomen). Previous work has demonstrated that four of the opisthosomal colour morphs (Yellow, Red front, Red blob and Red ring) are found in both sexes on Maui but on Hawai'i Yellow and Red blob are limited to females with the alleles controlling them producing morphs Red front and Red ring, respectively, in males. In addition, there is evidence for two unlinked loci controlling colour on Hawai'i, but only one on Maui. Here we use crosses between spiders of Maui and Hawai'i origin to examine further the differences in genetic structure between populations on the different islands. They also allow an assessment of the influence of a disrupted genetic background on the expression of colour morphs. Our results confirm the presence of two unlinked loci in Hawai'i. In addition, the control of expression of colour morphs in males and females is shown to be a property of the colour alleles themselves or of closely linked, cis-acting regulatory sequences. In all cases, the sex-limited morphs derived from Hawai'i behave on a mixed-island genetic background exactly as they do in pure Hawai'i crosses. Similarly, a mixed genetic background has no effect on the normal expression of the non-sex-limited morphs from both Hawai'i and Maui. The full expression of one male-limited morph from Hawai'i (Red front) is dependent on the presence of an X chromosome of Hawai'i origin. With one possible exception, dominance hierarchies are maintained on a mixed genetic background, possibly because for most morphs, dominance is determined by the superimposition of one pattern upon another. A preliminary model is developed to explain the quantum shift in the genetic control of the colour polymorphism in T. grallator on Hawai'i during its colonization from Maui.
\end{abstract}

Keywords: colour polymorphism, founder effect, Hawaii, sex-limitation, spider, Theridion grallator.

\section{Introduction}

In three previous papers (Gillespie \& Tabashnik, 1989; Oxford \& Gillespie, 1996a,b) we examined the genetic control of an extensive colour polymorphism in the Hawaiian happy-face spider, Theridion grallator (see plate 1 of Oxford \& Gillespie, 1996a). This species is endemic to four of the Hawaiian islands, Hawai'i itself, Maui, Moloka'i and O'ahu (Gon,

*Correspondence.

(C) 1996 The Genetical Society of Great Britain.
1985; Gillespie \& Tabashnik, 1990) where it occurs in wet to mesic native forest. The polymorphism includes characters of both the opisthosoma (abdomen) and carapace such that individual opisthosomal patterns are almost always associated with a particular carapace morph. This association is probably a consequence of control by different, but closely linked, loci rather than pleiotropic effects of alleles at one locus (Oxford \& Gillespie, 1996a).

Breeding data, and the distributions of morphs between the sexes in the wild, suggest that the 
genetic control of the opisthosomal polymorphism on Maui may be similar to that on Moloka'i (Oxford \& Gillespie, 1996a), but differs from that on Hawai'i (Oxford \& Gillespie, 1996b) in quite striking ways. On Maui, all the morphs appear to be determined by alleles at a single, major autosomal locus and are expressed equally in males and females; with one exception, dominance is determined by the superimposition of one pattern upon another. Yellow (unpatterned) is recessive to all patterned alleles. However, in populations on Hawai'i, certain morphs, phenotypically the same as on Maui, are sex-limited. Two pairs of morphs have been identified which act as if they are determined by single alleles differentially expressed in the two sexes. Yellow (females) and Red front (males) form one pair, and Red blob (females) and Red ring (males) the other. Yellow and Red front are the bottom recessive morphs within their appropriate sex. Within the constraints of sex-limited patterns, dominance hierarchies are the same as on Maui. The sex-limited morphs Red blob/Red ring act as if they are at the same locus as Red front + back, a morph which is not sex-limited. The Yellow/Red front allele may or may not be at this same locus. A further non-sex-limited morph, Black ring, is controlled by an allele at a locus unlinked to that determining Red front + back. There is no evidence for any of the loci being sex-linked.

In this paper we examine the inheritance of polymorphic patterns and their differential expression in males and females, in matings involving individuals from different islands and in backcrosses of the resulting progeny to parental stocks. This enables us to assess the importance of the genetic background in controlling phenomena previously investigated using within-island crosses.

\section{Materials and methods}

Spiders from Maui were sampled from the Nature Conservancy of Hawai'i's Waikamoi Preserve, near the common boundary of the Preserve and the Makawao State Forest, altitude $1360 \mathrm{~m}$. On Hawai'i spiders were collected from Thurston, Volcanoes National Park, altitude $1190 \mathrm{~m}$ and from Mawae (kipuka 8), Saddle Road, altitude $1600 \mathrm{~m}$. The Moloka'i individual derived from Pu'u Kolekole in the Nature Conservancy of Hawai'i's Kamakou Preserve, altitude $1110 \mathrm{~m}$. All collections were made in 1993 with the exception of the Saddle Road population, which was sampled in 1994.

Field techniques and laboratory rearing methods are described elsewhere (Oxford \& Gillespie, 1996a). The majority of spiders used here were isolated from the progeny of laboratory matings; a few were collected from the wild as immatures.

\section{Results}

The results of crosses are presented in Tables 1 and 2. Table 1 concerns the outcome of first-generation matings between individuals derived from different island populations and Table 2 details the results of crossing interisland progeny either back to parental stocks or inter se. Photographs of most of the morphs described here are to be found in Plate 1 of Oxford \& Gillespie (1996a).

\section{Genetics of opisthosomal morphs}

The first-generation interisland crosses are shown in Table 1. The first set of crosses conducted was to test the interaction between apparently identical morphs that are sex-limited on Hawai'i, but displayed in both sexes on Maui. Yellow females from Maui mated with Red front males from Hawai'i (no. 1, Table 1) produced broods in which all females were Yellow and all males were Red front, as did the reciprocal cross (no. 6) involving Yellow females from Hawai'i and Yellow males from Maui. These crosses demonstrate that the sex-limitation of Red front to males and Yellow to females, characteristic of the Thurston population, is also manifest on a mixed genetic background. They also emphasize the fact that all individuals from Thurston appear to be fixed for the Red front/ Yellow allele, because Yellow has been shown to be bottom recessive on Maui. There is one interesting difference between the outcome of the reciprocal crosses, however. Male progeny inheriting their sex-limited Red front allele from Hawai'i mothers (no. 6) showed a full, red-pigmented pattern (Plate 1b of Oxford \& Gillespie, 1996a). The equivalent morph derived from the reciprocal cross involving a Red front Hawai'i father (no. 1) in most cases exhibited much reduced red pigmentation (denoted as 'Rfr' in the tables). Indeed, the pigment often appeared as just one or two small dots, visible only in mature males and under a microscope. However, one male from cross no. 1 showed almost the full manifestation of the Red front pattern. The genetic background of the progeny of nos 1 and 6 (Table 1) is the same except for the source of the sex chromosomes, suggesting that sex-linked loci have some effect on the sex-limited expression of the Red front/Yellow allele. Although the chromosomes of $T$. grallator have not been investigated, the chromoso- 


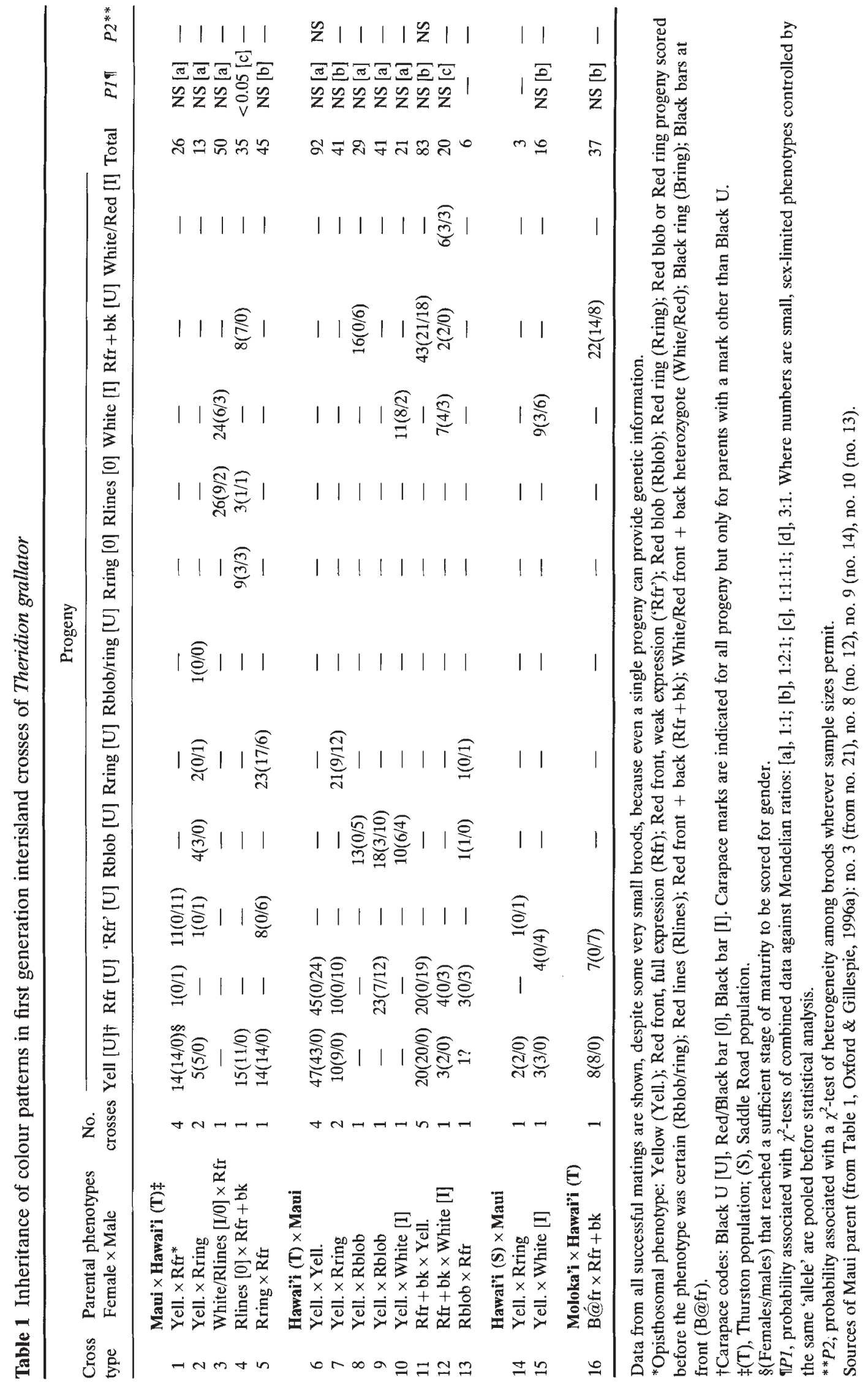




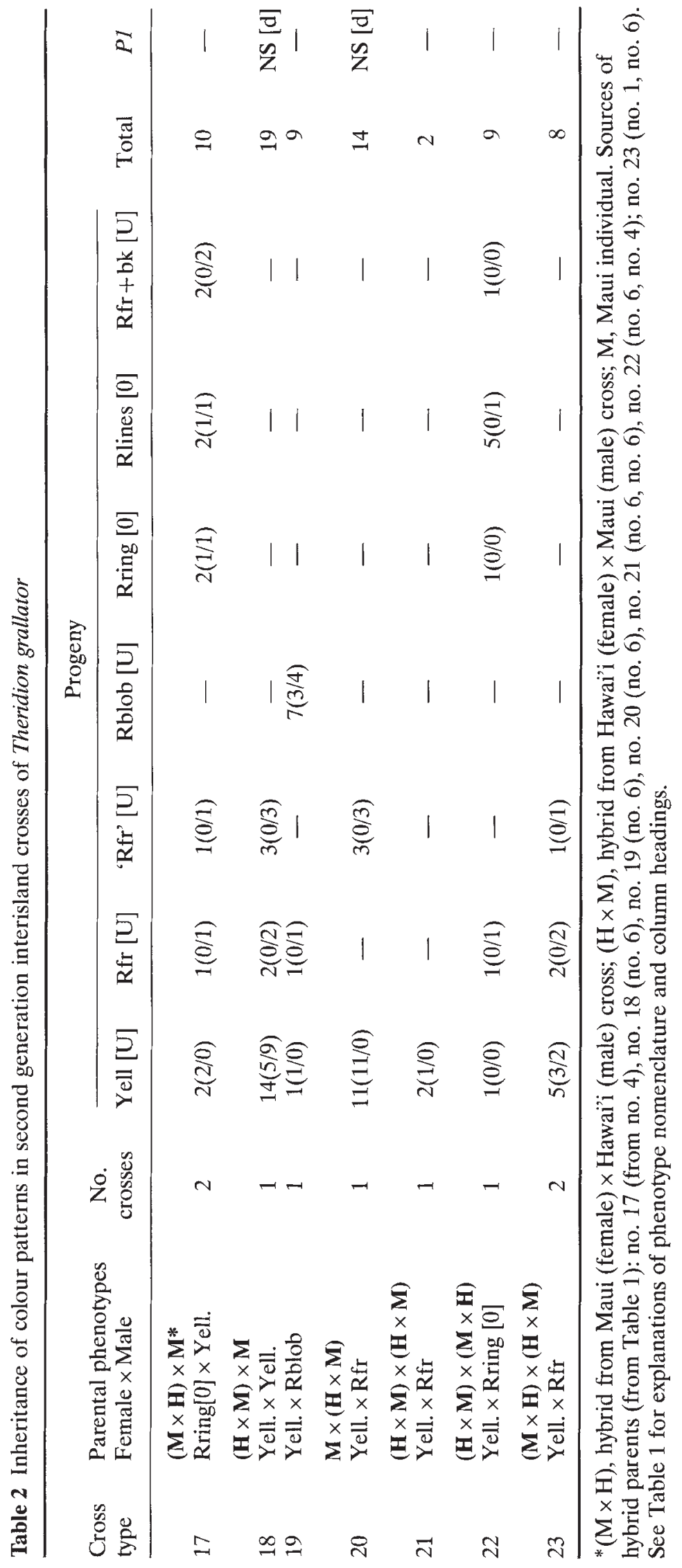


mal sex-determining mechanism in all theridiids examined to date is XXO (male) and XXXX (female) (e.g. Kageyama \& Seto, 1979; Tugmon et al., 1990), which essentially act as an XO, XX system (White, 1973). In general, full expression of the sex-limited Red front phenotype is manifest only when the male carries an X chromosome of Hawai' $\mathrm{i}$ origin. The same difference between reciprocal crosses was found in virtually all progeny of other matings from which the sex-limited Red front morph segregated (Table 1). The exception is the single male offspring from cross no. 14, which inherited an $\mathrm{X}$ chromosome from the Saddle Road population yet showed reduced pigmentation. The cross between a Moloka'i female with very heavy black bars at the anterior end of the opisthosoma and a Hawai'i male (no. 16, Table 1) produced offspring with the black bars, which made the degree of red pigmentation in Red front males difficult to categorize. Progeny of cross no. 15 were not scored for strength of pigment expression.

Reciprocal crosses were also made between two other morphs that are sex-limited on Hawai'i but not on Maui (Red ring and Red blob). Although numbers are small, crosses between Yellow and Red ring phenotypes (nos 2,5 and 7, Table 1) indicate that when the Red ring determinant came from Hawai'i (no. 2) it showed the same characteristics in the progeny as when on a pure Hawai'i genetic background (Oxford \& Gillespie, 1996b), producing Red ring males and Red blob females. When Red ring was introduced from Maui in either females (no. 5) or males (no. 7) it was not sex-limited and segregated with the Yellow/Red front sex-limited patterns inherited from the Hawai'i parent. The final cross involving morphs that are sex-limited on Hawai'i used Red blob phenotypes from both Hawai'i and Maui. When inherited from a Maui parent (nos 8 and 9, Table 1) Red blob was not sex-limited, whereas when this morph came from the Hawai'i parent (no. 13, Table 1) it was expressed as Red ring in males and as Red blob in females, although numbers of progeny are again small.

The expression of a particular morph is therefore characteristic of the population from which it derived, irrespective of the genetic background. Different outcomes from reciprocal crosses indicate that whatever is controlling the sex-limited expression of alleles is either a property of the allele itself or of a tightly linked regulatory locus. If the latter is the case, the effects of the regulatory elements are cis-acting. The one cross involving a female from Moloka'i (no. 16) showed that here too, sex-limitation characteristic of Hawai'i populations is main- tained on a mixed-island genetic background.

A second set of crosses was conducted to examine the allelism of pairs of patterned morphs within the Maui population. Thus, White and Red lines (no. 3), Red blob and Red front + back (no. 8), Red blob and Red front (no. 9) and Red blob and White (no. 10) are, respectively, shown to segregate as alleles at one major locus. The progeny phenotypes and ratios are those expected given the sources of the patterned parents (see last footnote to Table 1). These observations support previous conclusions regarding the allelic nature and dominance hierarchy of colour morphs on Maui (Oxford \& Gillespie, 1996a).

Matings involving the Hawai'i Red front + back phenotype are worthy of note. Cross no. 11 (Table 1) gave large numbers of progeny segregating in a 1:1:2 ratio of Yellow (females):Red front (males): Red front + back (females/males), indicating that the patterned parents were either heterozygous for Red front + back and Yellow/Red front alleles or that they were heterozygous for Red front + back and a bottom recessive allele, perhaps Yellow, at one locus and homozygous for the Yellow/Red front allele at a different locus (discussed more fully below). As noted above, sex limitation, or the lack of it, on the mixed genetic background is the same as in pure Hawai'i crosses. The brood of cross no. 4 (Table 1) between Red lines and Red front + back was unusual in that it was heavily female-biased and phenotype frequencies differed significantly from a 1:1:1:1 ratio. However, it did show that the Red ring morph can be produced by the combination of Red lines and Red front + back on a mixed genetic background, as was found within Maui crosses (Oxford \& Gillespie, 1996a). Finally, cross no. 12, involving Red front + back and White, demonstrated an apparent breakdown of dominance. Some White individuals showed variable amounts of red pigment (the White/Red phenotype in Table 1) deposited at the anterior end of the opisthosoma and, to a lesser extent, in places along the black rim around the white shield (see Plate 1h of Oxford \& Gillespie (1996a) for a normal White phenotype). The one similar cross made within Maui showed complete dominance of White over Red front + back (Oxford \& Gillespie, 1996a).

Table 2 provides information on second generation crosses between interisland hybrids. The first set of crosses focused on the sex-limited Maui-Hawai'i hybrids. Cross no. 18, between an inter-island Yellow female (from no. 6, Table 1) and a Yellow male from Maui, yielded offspring in a good 3:1 ratio of Yellow:Red front. The former comprised both males and females, whereas the latter were all 
males, two with a full Red front (Rfr) and three with much reduced red pigmentation ('Rfr'). These results are most simply explained with a model in which it is assumed that the sex-limited Red front/ Yellow morph from Hawai'i and the Yellow morph from Maui are controlled by alleles at the same locus, and that a Hawai'i X chromosome is required for full expression of Red front in males. The reciprocal cross (no. 20), involving a male from no. 6 (Table 1), should give the same 3:1 ratio but patterned male offspring would be expected to have a weak Red front because all will inherit an $\mathrm{X}$ chromosome from their Maui mother. This was indeed found but Yellow males were not recovered. On the basis of the model described above, cross no. 23 , between two interisland 'hybrids' (females from no. 1 and males from no. 6 , Table 1), is expected to yield a ratio of Yellow females:Yellow males:full Red front males:weak Red front males of 8:2:3:3. Assuming a 1:1 sex ratio, this gives an overall phenotype ratio of Yellow:Red front of 5:3, which is exactly that shown in the rather small brood.

A second set of crosses examined interactions between morph patterns. Two crosses in Table 2, nos 17 and 22, concern the Red ring phenotype (from no. 4, Table 1). Numbers offspring are again very small but in both sets of progeny Red lines and Red front + back segregated, confirming that Red ring can be produced by the combination of these two patterned morphs. Consider in more detail cross no. 17. The female parent was shown to possess both the Red lines and the Red front + back determinants. If these are controlled by alleles at one locus, the progeny of this cross should segregate in a simple 1:1 ratio for the two patterned morphs. However, this is not the case as Yellow females, Red front males and the Red ring phenotype also appear in the offspring; Red lines and Red front +back cannot therefore be allelic to one another.
Neither can the Red front + back and the sex-limited Red front/Yellow morphs be allelic because, in the construction of the Red ring female parent of no. 17, the Red lines determinant came from her Maui mother and both the Red front/Yellow and the Red front + back determinants must have derived from her Hawai'i father. The simplest model to explain this situation assumes two unlinked loci. In the Red ring parent of no. 17, one locus is heterozygous for Red lines and Red front/Yellow determinants and the other heterozygous for Red front + back and Yellow determinants (see Fig. 1). It is also assumed that the Yellow determinant can be present at both loci and that the Yellow male parent from Maui was homozygous. This leads to the prediction of Red lines:Red ring:Red front + back:Yellow females: full Red front males:weak Red front males in the ratio 4:4:4:2:1:1. Neither no. 17 nor no. 22 is inconsistent with this interpretation.

\section{Genetics of carapace morphs}

All three major carapace morphs from Maui, Black $\mathrm{U}$, Black bar and Red/Black bar, have been introduced onto a mixed Hawai'i/Maui genetic background (Table 1). The dominance relationships among them are exactly as was found in crosses within Maui, namely Black bar and Red/Black bar are both fully dominant to Black $U$ (nos 3, 4, 10 and 12, Table 1; nos 17 and 22, Table 2) (Oxford \& Gillespie, 1996a). Cross no. 3 confirms that Black bar and Red/Black bar are controlled by alleles at one locus on Maui.

\section{Discussion}

The results of the crosses presented here demonstrate that, although there are major differences in the genetical control of the colour polymorphism in
Locus I
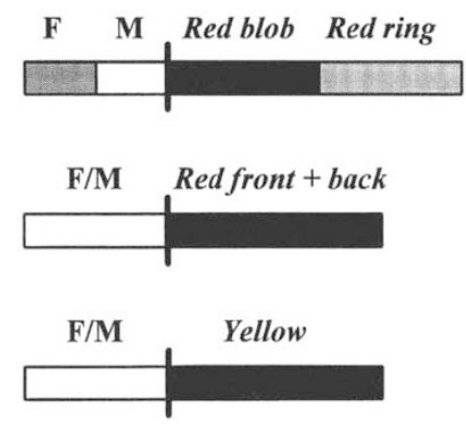

Locus II
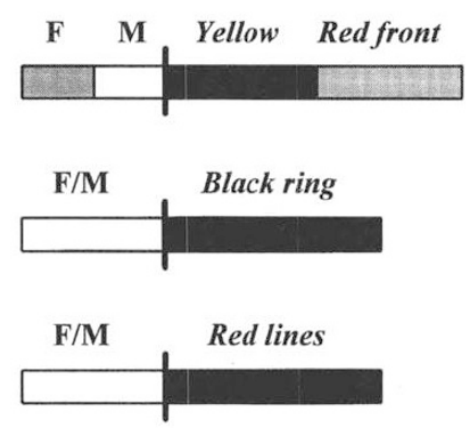

Fig. 1 Possible model of the genetic control of the colour polymorphism in Theridion grallator on Hawai'i. Putative alleles present at each of two unlinked loci are shown with the complex, sex-limited 'alleles' first. $\mathbf{F}$ and $\mathbf{M}$ refer to regulatory sequences activated in females and males, respectively. $F$ switches on the first of the tandem structural alleles while $M$ switches on the second. $\mathrm{F} / \mathrm{M}$ refers to regulatory sequences that are not sex-sensitive. All colour alleles are from Hawai'i with the exception of Red lines allele from Maui. See text for more details. 
T. grallator on Maui and on Hawai'i, the populations have not differentiated so much that gene exchange between them is impossible. Not only are the hybrids between islands viable, they are also fertile. The apparently lower brood size in crosses involving hybrids (Table 2) compared to first generation interisland (Table 1) and within-island crosses (Oxford \& Gillespie, 1996a,b) is probably an artifact. Initial scores for crosses in Table 2 were made at an early stage of development before the morphs of some individuals were certain. Later scores were made of penultimate instar spiders and these are the ones presented here. If numbers of offspring in the broods when first counted are considered, they are lower, but not significantly so, than the brood sizes of both laboratory and wild-mated females from within Maui or Hawai'i.

Matings between individuals from Maui and Hawai'i produced progeny with the same phenotypic colour-morph characteristics as would have been produced in within-island crosses. Thus, whether a morph is sex-limited or not seems to be a property of the morph itself rather than an interaction involving the wider genetic background. That other elements of the genetic background can be important under certain conditions is demonstrated by the male-limited Red front morph from Hawai'i, which is only expressed fully in the presence of an X chromosome of Hawai'i origin (the apparent exceptions in Table 1, single males in progenies of crosses nos 1 and 14, may be real or a result of misclassification). It appears, then, that modifiers on the Hawai' $i$ $\mathrm{X}$ chromosome are required for complete expression of the Red front morph or, alternatively, that a locus (or loci) on an $\mathrm{X}$ chromosome of Maui origin suppresses expression to some extent. If the latter is the case, it appears to have no effect on the Red front morph of Maui origin. The degree of expression of Red front extends both to the red pigmentation itself and to the underlying guanine deposits (Oxford \& Gillespie, 1996a). Dominance is also relatively unaffected in interisland crosses. Only the interaction of White and Red front + back (no. 12, Table 1) suggests that the inhibitory effect of the White morph on overlying red and black pigmentation, which on Maui is usually complete, may be less so on an interisland genetic background. However, only one cross generating this particular heterozygote has been made within Maui so conclusions must remain tentative.

The lack of dominance breakdown in interisland crosses contrasts with numerous other cases of colour polymorphisms in which it has been shown that the genetic background is vital for the manifes- tation of full dominance. For example, Ford (1955) showed that in Triphaena [= Noctua] comes (the lesser yellow underwing moth) on the Outer Hebrides and Orkney island groups (Scotland), the almost complete dominance of the dark curtisii over the pale comes form breaks down in interisland crosses. Ford argued that the dominance of curtisii over comes has evolved independently in the two populations as a result of selection for different modifier elements. Similar conclusions were drawn by Clarke and Sheppard (1960) who made interpopulation crosses using the mimetic swallowtail butterfly Papilio dardanus. The lack of such an effect in $T$. grallator might be a result of (i) the relatively short time the Maui and Hawai'i populations have been separated (maximum $0.4 \mathrm{Myr}$, the age of the oldest land on Hawai'i Island), so that genetic backgrounds have differentiated to only a small extent, and/or (ii) the fact that dominance in this case seems to be produced simply by the superimposition of one pattern upon another. It is interesting to note that the morph associated with the breakdown of dominance, White, is the only one in which the mechanism of dominance is not by superimposition but by suppression of pigmentation. Further intraand interisland crosses are required to ascertain whether the reduced inhibition in this morph is found only on a mixed genetic background. In this context it will be important to test whether crosses between populations from more distant islands, e.g. Hawai'i and O'ahu, behave differently.

The cosegregation of specific alleles with their sex-limited or non-sex-limited control parallels the situation described for a colour polymorphism in Enoplognatha ovata, another Theridiid spider (Oxford, 1983). The regulatory elements are presumably integral parts of the colour alleles themselves or are tightly linked sequences. Whatever the details, it is clear from interisland crosses that the control elements are cis-acting on their respective colour alleles. It is possible that the non-sex-limited condition is the default and that sex-limitation results from the integration of new sequences into the control region of certain colour alleles on Hawai'i such that an equivalent sequence is absent from non-sex-limited alleles. Alternatively, the control elements might be the same but mutant sequences on Hawai'i respond only to particular sex-specific cues that are not used on Maui.

Differences between populations of a species with regard to whether particular colour morphs are sex-limited or not have been reported in a number of cases. In the spider $E$. ovata, for example, the top dominant ovata morph is equally represented in 
both sexes in Britain (Oxford, 1983, 1985) and in North America (Reillo \& Wise, 1988; Reillo, 1989), but in at least some continental European populations this morph is sex-limited to females (Gerhardt, 1921; Hippa \& Oksala, 1979). Oxford (1983) speculated that the recombinant chromosome containing the ovata structural allele and the sex-limiting 'late' regulatory allele was not present in Britain. Similarly, in Philaenus spumarius, the meadow spittlebug, certain melanic morphs are sex-limited to females over much of continental Europe, whereas others are found in both sexes but at much lower frequencies in males (summarized by Stewart \& Lees, 1988 and Halkka \& Halkka, 1990). Males have been found in Europe with the female-limited patterns, but they are exceedingly rare. Stewart \& Lees (1987, 1988), however, reported that in Britain the total frequency of melanic morphs is not significantly different between the sexes in most populations. Melanic phenotypes sex-limited to females in Europe are also regularly found in males, albeit at lower frequencies than in females. The very rare breakdown of sex-limitation in $P$. spumarius in Europe is interesting in that it parallels the discovery of a small number of Red front female $T$. grallator in the Saddle Road population on Hawai'i (Oxford \& Gillespie, 1996b).

The genetic basis of the colour polymorphism differs markedly between $T$. grallator populations on Maui and Hawai'i. The relative ages of the islands (Funk \& Wagner, 1995) suggest that colonization would almost certainly have occurred in the direction of Maui to Hawai'i. Because the sex limitation of at least Red front and Yellow is island-wide on Hawai'i, it appears that the genetic differences between the islands probably arose during or soon after the founding event. It is clearly desirable to incorporate all the information we have to date into a tentative genetic model so that we can explore the sorts of genomic changes that might have occurred during the colonization of Hawai'i. In a previous paper (Oxford \& Gillespie, 1996b), we demonstrated that at least two unlinked loci are involved in the control of the colour polymorphism on Hawai'i, with Red front + back and Black ring alleles located at different loci. Here, cross no. 17 provides clear evidence that Red front + back and Red lines cannot be at the same locus, and also that the sex-limited Red front/Yellow cannot be at the same locus as Red front + back. Segregation ratios within the offspring of no. 17 suggest that the loci concerned are not linked. Matings within Hawai'i (Oxford \& Gillespie, 1996b) showed that Red front + back and the sex-limited Red blob/Red ring segregate as if they are controlled by alleles at the same locus. Putting these observations together yields a minimal model with two unlinked loci or groups of loci (Fig. 1). An important phenomenon to explain is how pairs of patterns that are controlled by multiple alleles at one major locus on Maui can apparently be determined by the differential expression of single alleles on Hawai'i. The Red front/Yellow situation could be a result of a sex-hormone-mediated switching on or off of a Red front allele (with Yellow representing a 'null' pattern produced by another, precursor, locus), as seems to be the case in E. ovata (Oxford, 1983). The Red blob/Red ring pair is more difficult to explain in this way because this time the morph that is limited to males is less patterned than the female equivalent, but whatever mechanism is responsible it seems likely to be the same for both pairs of sex-limited morphs.

Figure 1 presents a first attempt at a model to explain the control of the colour polymorphism on Hawai'i. It is suggested that the sex-limited patterns are a result of tandem structural alleles controlled by a regulatory sequence at least part of which is sensitive to sex hormones or cell sex-specific transcriptional regulators [see, for example, Carlson \& Botstein (1982) for a broadly similar model]. In females the regulatory sequence activates the first of the structural alleles whereas in males it is the second structural allele that is switched on. Alternatively, differential splicing of structural gene transcripts in males and females could also produce the observed pattern (e.g. Baker, 1989; McKeown, 1992). This disposition and control of structural loci would explain why in males the more dominant Red front (of the Red front/Yellow pair) is activated whereas for the Red blob/Red ring pair of morphs, the more recessive is expressed in males. The complex alleles for the two sex-limited morph pairs are at different, unlinked loci, as discussed above. Crossing data suggest that Red front + back is allelic to Red blob/Red ring at locus I and the fact that some morphs controlled by locus II (Red front/ Yellow) are recessive to Red front + back means that locus I must also contain a bottom recessive allele (Yellow) or a 'null' allele if the basic yellow background colour is determined by yet another locus. Locus II houses the Red front/Yellow sex-limited allele complex and possibly the allele for Black ring. Red lines from Maui could also be determined at locus II, but not at locus I.

The presence of complex sex-limited alleles at two unlinked loci poses a problem in terms of the evolution of this system from the apparently single, major locus situation on Maui. It seems likely that the 
sex-limited alleles were created by some sort of local chromosomal rearrangement prior to loci I and II becoming separated by a translocation or inversion event. Duplication of loci may not be involved because there is circumstantial evidence that the single 'locus' on Maui may, in fact, be a supergene consisting of two or more closely linked loci (Oxford \& Gillespie, 1996a,b). If the different carapace morphs (Oxford \& Gillespie, 1996a) are indeed controlled by a locus tightly linked to that/those for the opisthosomal morphs, as seems very likely, then this information too must be incorporated into future models. Clearly, further work on this unique evolutionary system is required in order to gain a fuller understanding of the possible influence of founder events on characters controlled by major loci (see, for example, McDonald, 1989, 1995).

\section{Acknowledgements}

The work reported here was supported in Hawai'i by National Science Foundation Grant DEB 9207753 to R.G.G and G.S.O., and in York by Natural Environment Research Council Grant GR9/1530 to G.S.O. Additional support, which enabled G.S.O. to work in Hawai'i during 1993, is gratefully acknowledged from the British Ecological Society, the Royal Society, the Bonhote Bequest, the Percy Sladen Memorial Fund and the University of York. We thank Peter Follett, Roma Oxford, David Preston, George Roderick and An-Ming Tan for assistance in the field, and Patrick Conant, Linden Doescher, Kelvin Kanegawa and Bruce Tabashnik for supplying diamond-back moths, Drosophila grimshawi and D. melanogaster as prey. The late Keith Partridge provided essential logistical support in York. For assistance in gaining access to collecting sites we would like to thank the Nature Conservancy of Hawai'i, in particular Ed Misaki (Kamakou Preserve, Moloka'i), Paul Higashino and Mark White (Waikamoi Preserve, East Maui) and the National Parks Service (Thurston, Hawai'i). Thanks also to Bill Mull and Sam Gon for discussion and to John Sparrow and two anonymous referees for comments on the manuscript.

\section{References}

BAKER, B. s. 1989. Sex in flies: the splice of life. Nature, 340, 521-524.

CARLSON, M. AND BOTSTEIN, D. 1982. Two differentially regulated mRNAs with different $5^{\prime}$ ends encode secreted and intracellular forms of yeast invertase. Cell,
28, $145-154$.

CLARKE, C. A. AND SHEPPARD, P. M. 1960. The evolution of dominance under disruptive selection. Heredity, 14, 73-87.

FORD, E. B. 1955. Polymorphism and taxonomy. Heredity, 9, 255-264.

FUNK, V. A. AND WAGNER, w. L. 1995. Biogeographic patterns in the Hawaiian Islands. In Wagner, W. L. and Funk, V. A. (eds) Hawaiian Biogeography: Evolution on a Hot Spot Archipelago, pp. 379-419. Smithsonian Institution Press, Washington.

GERHARDT, U. 1921. Vergleichende Studien über die Morphologie des Männlichen Tasters und die Biologie der Kopulation der Spinnen. Arch. Naturgesch., 87, 78-247.

GILLESPIE, R. G. AND TABASHNIK, B. E. 1989. What makes a happy face? Determinants of colour pattern in the Hawaiian happy face spider Theridion grallator (Araneae, Theridiidae). Heredity, 62, 355-363.

GILLESPIE, R. G. AND TABASHNIK, B. E. 1990. Maintaining a happy face: stable colour polymorphism in the spider Theridion grallator (Araneae, Theridiidae). Heredity, 65, 67-74.

GON, S. M. 1985. Comparative Behavioral Ecology of the Spider Theridion grallator (Simon) (Araneae: Theridiidae) in the Hawaiian Archipelago. Ph.D. Thesis, University of California, Davis, CA.

HALKKA, O. AND HALKKA, L. 1990. Population genetics of the polymorphic meadow spittlebug Philaenus spumarius (L.). Evol. Biol., 24, 149-191.

Kageyama, A. AND Seto, T. 1979. Chromosomes of seven species of Japanese theridiid spiders. Chromosome Inf. Serv., 27, 10-11.

McDONALD, J. F. 1989. The potential evolutionary significance of retroviral-like transposable elements in peripheral populations. In Fontdevila, A. (ed.) Evolutionary Biology of Transient Unstable Populations, pp. 190-205. Springer-Verlag, New York.

McDONALD, J. F. 1995. Transposable elements: possible catalysts of organismic evolution. Trends Ecol. Evol., 10, $123-126$.

Mckeown, M. 1992. Alternative mRNA splicing. Ann. Rev. Cell. Biol., 8, 133-155.

OXFORD, G. S. 1983. Genetics of colour and its regulation during development in the spider Enoplognatha ovata (Clerck) (Araneae: Theridiidae). Heredity, 51, 621-634.

OXFORD, G. s. 1985. Geographical distribution of phenotypes regulating pigmentation in the spider Enoplognatha ovata (Clerck) (Araneae: Theridiidae). Heredity, 55, 37-45.

OXFORD, G. S. AND GILlESPIE, R. G. 1996a. Genetics of a colour polymorphism in Theridion grallator (Araneae: Theridiidae), the Hawaiian happy-face spider, from Greater Maui. Heredity, 76, 238-248.

OXFORD, G. S. AND GILlESPIE, R. G. 1996b. Quantum shifts in the genetic control of a colour polymorphism in Theridion grallator (Araneae: Theridiidae), the Hawaiian happy-face spider. Heredity, 76, 249-256.

REILlo, P. R. 1989. Color polymorphism in the spider 
Enoplognatha ovata (Araneae; Theridiidae): broad-scale morph-frequency variation in northeastern North America. Am. Midl. Nat., 122, 199-203.

REILLO, P. R. AND WISE, D. H. 1988. Genetics of color expression in the spider Enoplognatha ovata (Araneae; Theridiidae) from Coastal Maine. Am. Midl. Nat., 119, 318-326.

STEWART, A. J. A. AND LEES, D. R. 1987. Genetic control of colour polymorphism in spittlebugs (Philaenus spumarius) differs between isolated populations. Heredity, 59, 445-448.
STEWART, A. J. A. AND LEES, D. R. 1988. Genetic control of colour/pattern polymorphism in British populations of the spittlebug Philaenus spumarius (L.) (Homoptera: Aphrophoridae). Biol. J. Linn. Soc., 34, 57-79.

TUGMON, C. R., BROWN, J. D. AND HORNER, N. V. 1990. Karyotypes of seventeen USA spider species (Araneae, Araneidae, Gnaphosidae, Loxoscelidae, Lycosidae, Oxyopidae, Philodromidae, Salticidae and Theridiidae) J. Arachnol., 18, 41-48.

white, M. J. D. 1973. Animal Cytology and Evolution, 3rd edn. Cambridge University Press, Cambridge. 